\title{
The Accuracy of Barcode Sign in Diagnosis of Pneumothorax Associated with Acute Respiratory Failure in Emergency Department
}

\author{
Mohamed Elbahnasawy ${ }^{1 *}$, Ghada Atef Attia ${ }^{2}$, \\ Atef Hamad Teima ${ }^{3}$ and Abdel Hady Mohamed Taha ${ }^{4}$ \\ ${ }^{1}$ Department of Emergency Medicine and Traumatology, ${ }^{2}$ Department of Chest Disease, \\ ${ }^{3}$ Department of Radiodiagnosis, ${ }^{4}$ Department of Cardiothoracic Surgery, \\ Tanta University Faculty of Medicine, Egypt
}

*Corresponding author

\section{Keywords}

Lung ultrasound;

Emergency;

Pneumothorax

Article Info

Accepted:

10 May 2019

Available Online:

10 June 2019

\section{A B S T R A C T}

The objective of this study was identifying the accuracy of barcode sign using lung ultrasound in diagnosing pneumothorax presented to Emergency department with acute respiratory failure. This was cross sectional observational study conducted between March 2017 and March 2018 on patients 16 patients older than 18 years suspected to have pneumothorax presented to Emergency department with acute respiratory failure who were admitted to ICU. Patients were excluded retrospective from the study if there were multiple etiologies of respiratory failure. All patients subjected to lung ultrasound examination by emergency physician who not involved in the management of the patients. Lung ultrasound diagnosis was compared with the final ICU diagnosis as the gold standard. The specificity, sensitivity, positive predictive value (PPV) and negative predictive (NPP) of pulmonary ultrasound in diagnosis pneumothorax as compared with final ICU diagnosis were $83.3 \%, 100 \%, 100 \%$ and $66.7 \%$ respectively lung ultrasound can be used with high accuracy in diagnosis of pneumothorax in Emergency Department.

\section{Introduction}

Patients presented to the Emergency Department with acute respiratory failure remain a big challenge for diagnosis to emergency physicians. They need urgent care and rapid diagnosis to guide treatment.(1)

The conventional methods for diagnosis pneumthorax in Emergency department are chest $\mathrm{x}$-ray, CT scan. However, they are time consuming, in addition to the hazards of ionizing radiation(2). In critically ill patients presented to the Emergency Department, it is even harder to obtain supine $\mathrm{x}$-ray and the quality of portable $\mathrm{x}$ ray is usually not good to interpret.(3)

Lung ultrasound is easy to learn, bed side, fast and point of care. Point of care ultrasound is increasingly used by many clinicians, as it is fast, bedside and noninvasive, helping the 
physicians to make fast and accurate decision about their patients in acute setting (4, 5). Lung ultrasound is a useful tool for detecting many lung pathologies and guiding emergency medicine physicians to get through different disease that need urgent interference such as pulmonary edema, pleural effusion and pneumonia $(6,7)$. The accuracy of lung ultrasound in patients with acute respiratory failure is high since LUS can detect lung aeration changes in many lifethreatening conditions, such as congestive heart failure, acute respiratory distress syndrome, pneumothorax and pneumonia (8, 9)

The primary objective of this study is to evaluate the accuracy of lung ultrasound by emergency physician who are not expert in using ultrasound in diagnosis of pneumothorax presented to emergency department with acute respiratory failure.

\section{Patients and Methods}

This was a cross-sectional observational study conducted in Tanta University Emergency Hospital from March 2017 to end of February 2018 on 16 patients presented to Emergency medicine department with acute respiratory failure (ARF) and admitted to intensive care unit. The study was approved by the Research Ethics Committee of Tanta University Faculty of Medicine.

\section{Study setting and population}

All adult patients (>18 years) presenting to the emergency department with acute respiratory failure defined as respiratory rate more than 22 and oxygen saturation less than $90 \%$ and or $\mathrm{PaO}_{2}$ less than $60 \mathrm{mmHg}, \mathrm{PaCO}_{2}$ more than $50 \mathrm{mmHg}$. Patients who met one of the following criteria were excluded retrospectively: patients with multiple diagnosis, sis.

\section{Study protocol}

Patients had lung ultrasound by emergency physician who did not participate in the patient's management, undertaken by other emergency physicians. All the participating Emergency physicians received lung ultrasound training for 6 hours ( 3 hours theory and 3 hours practical) prior the start of the study.

A written informed consent was obtained from the patients or their relatives. The patient or his relative received an explanation on the purpose of the study and patient had a secret code number to ensure privacy to participants and confidentiality of the data.

Pulmonary ultrasonography was performed using Digital Ultrasonic Imaging System Model Phillips Affiniti 50G and portable Mindray dp20.

Examination of the patients was done using linear transducer. Patients were investigated in a semi-recumbent position, or supine if intubated. Scans will be longitudinal. The pleural lines that will be seen between two rib shadows indicate the pleural layers. Each hemithorax was examined at 6 points and the images were saved to be later interpreted.

After examination of the patients the transducer was cleaned with antiseptic agent. Diagnosis of pneumothorax was made when barcode or stratosphere sign is documented.

\section{Outcome measures}

The final intensive care diagnoses were used as the gold standard. The ICU team were blinded to LUS diagnosis.

The sensitivity, specificity, and likelihood ratios and their 95\% confidence intervals were calculated. 


\section{Results and Discussion}

During the period from March 2017 to March 2018, 16 patients were enrolled in the study. All patients were admitted to intensive care unit. In this study, the age of patients ranged from 25 to 80 with mean age about 56.39 years. $60 \%$ of patients included in our study were males and $40 \%$ were females as shown in table 1.

Accuracy of absent lung sliding and barcode sign using $\mathrm{M}$ mode by pulmonary ultrasound in diagnosis of pneumothorax as compared with final ICU diagnosis revealed that it had true positive diagnosis in 10 patients, false negative in 2 patients, true negative in 4 patients and false positive in no patients when compared with the final ICU diagnosis.

The specificity, sensitivity, positive predictive value (PPV) and negative predictive (NPP) of pulmonary ultrasound in diagnosis pneumothorax as compared with final ICU diagnosis were $83.3 \%, 100 \%, 100 \%$ and $66.7 \%$ respectively.

In this study barcode sign using linear probe with $\mathrm{M}$ mode is used in diagnosing pneumothorax in emergency department. It donates lung sliding that will be absent in any condition in which the pleura is either not directly opposed (pneumothorax, effusion), are stuck together (pneumonia, ARDS, pleurodesis), or in absent respiration (pneumonectomy, one lung intubation). The appearance of this on M-mode is horizontal straight lines - the 'stratosphere sign' (also termed barcode sign) (Figure 1 and 2).

It was found that it has a great accuracy in diagnosing pneumothorax in critically ill patients in emergency department suspected to have pneumothorax. In agreement with our study, Abdalla W et al., in 2016 studied the accuracy of absent lung sliding using pulmonary ultrasound for detection of pneumothorax as compared with CT chest as a gold standard and found that sensitivity, specificity were $86.1 \%$, $97.4 \%$ respectively ${ }^{(10)}$.

Also in agreement with our study Mumtaz et al., (2016) compared the diagnostic accuracy of absent lung sliding by bedside ultrasound and supine chest radiography for the diagnosis of traumatic pneumothorax using CT chest as the gold standard and found that sensitivity, specificity of ultrasound were $83.33 \%, 100 \%$ respectively ${ }^{(11)}$.

The results of this study were in line with Zhang M et al., (2006) that included 135 patients and aimed to detect and assess the size of the PTX in polytraumatized patients using bedside ultrasonography. The diagnostic sensitivity, specificity, PPV and NPV for ultrasonography were $86.2 \%, 97.2$ $\%,{ }^{(12)}$

In Contrary with this study, Rowan KR, et al., (2002) compared the accuracy of thoracic ultrasonography with accuracy of supine chest X-Ray in the detection of traumatic pneumothoraxes on 27 patients with blunt thoracic trauma. Results showed that sensitivity and specificity of thoracic ultrasonography were 100 and $93.8 \%$ respectively. ${ }^{(13)}$ This because they depend on founding lung point that is pathognomonic for pneumothorax. And in this study, absence of lung sliding was used to diagnose pneumothorax. Absence of lung sliding can be caused by other pathologies such as severe atelectasis, main stem bronchus intubation. Presence of lung sliding can exclude pneumothorax, but its presence is highly suggestive of pneumothorax. ${ }^{(9)}$

\section{Limitations of the study}

This study was single center study with small size sample. We may need another study to compare the time lag between using lung 
ultrasound and other methods such as CT scan or chest $\mathrm{x}$ ray and its affection on the prognosis of the patient presented to
Emergency room with acute respiratory failure.

Table.1 General characteristics of the patients

\begin{tabular}{|l|l|l|}
\hline Parameter & Range & Mean and SD \pm \\
\hline Age & $\mathbf{2 5 - 7 0}$ & $56.39 \pm \mathbf{1 7 . 5 5}$ \\
\hline Gender & $\mathbf{6 0 \%}$ male, 40 \% female \\
\hline MAP $(\mathbf{m m H g})$ & $45-109$ & $79.19 \pm 19.67$ \\
\hline HR & $69-123$ & $87 \pm 8.17$ \\
\hline RR & $18-33$ & $25.97 \pm 4.1$ \\
\hline SPO2 (\%) & $74-90$ & $83.08 \pm 3.03$ \\
\hline PaO $_{2}$ & $44-75$ & $56 \pm 8.16$ \\
\hline PaCO2 & $29-70$ & $44 \_ \pm 13.5$ \\
\hline APACHE II SCORE & $4-25$ & $12.2 \pm 5.39$ \\
\hline PH & $7.10-7.42$ & $7.200 \pm 0.704$ \\
\hline Temp & $36^{\circ}-39.4^{\circ}$ & $37.5^{\circ} \pm 0.37^{\circ}$ \\
\hline
\end{tabular}

$\mathrm{MAP}=$ Mean arterial pressure $\mathrm{HR}=$ hear rate $\mathrm{RR}=$ respiratory rate

APACHE II SCORE=acute physiology and chronic health evaluation.

Fig.1 shows barcode sign using M mode by superficial probe

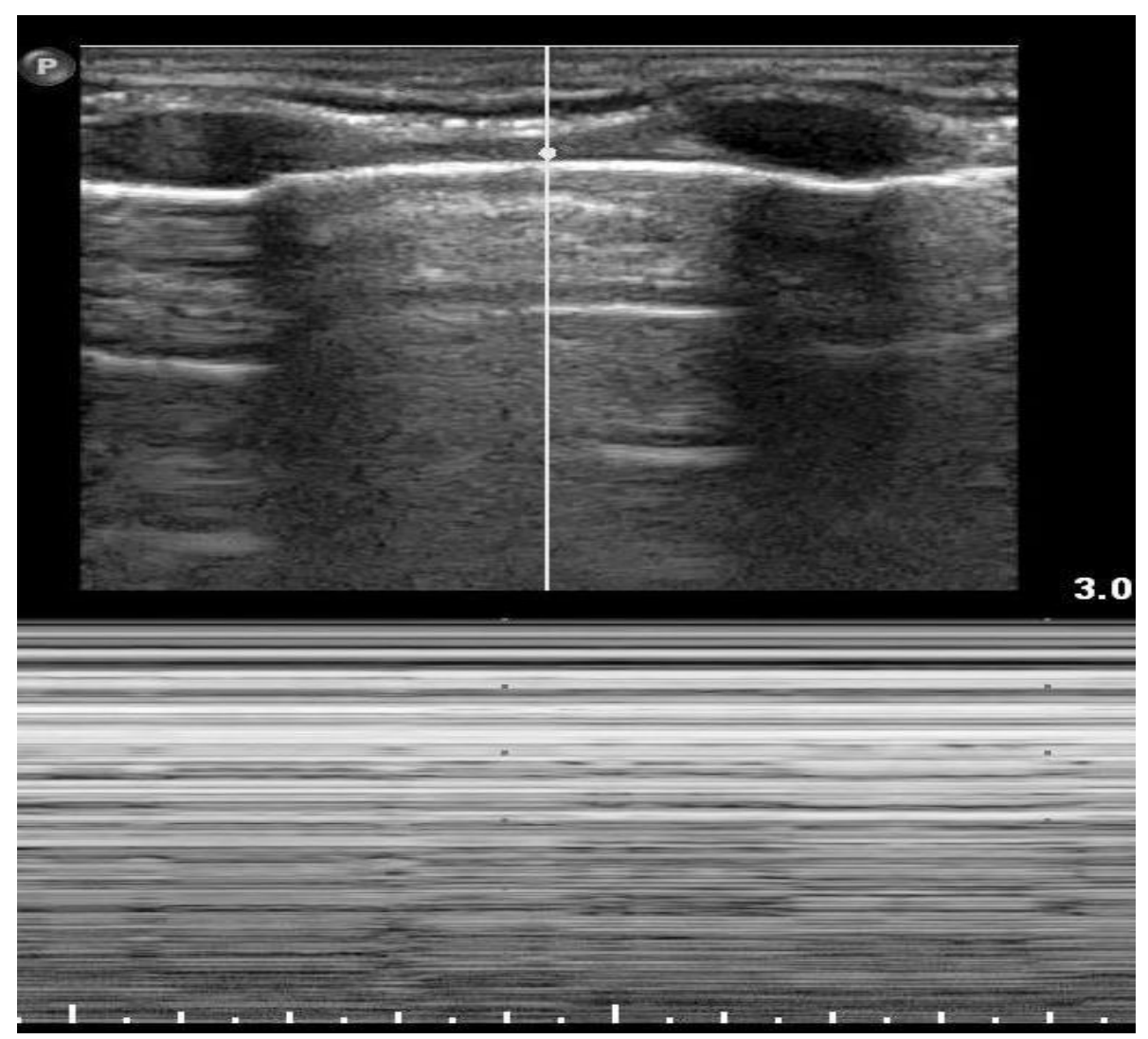


Fig.2 showing stratosphere sign or barcode sign

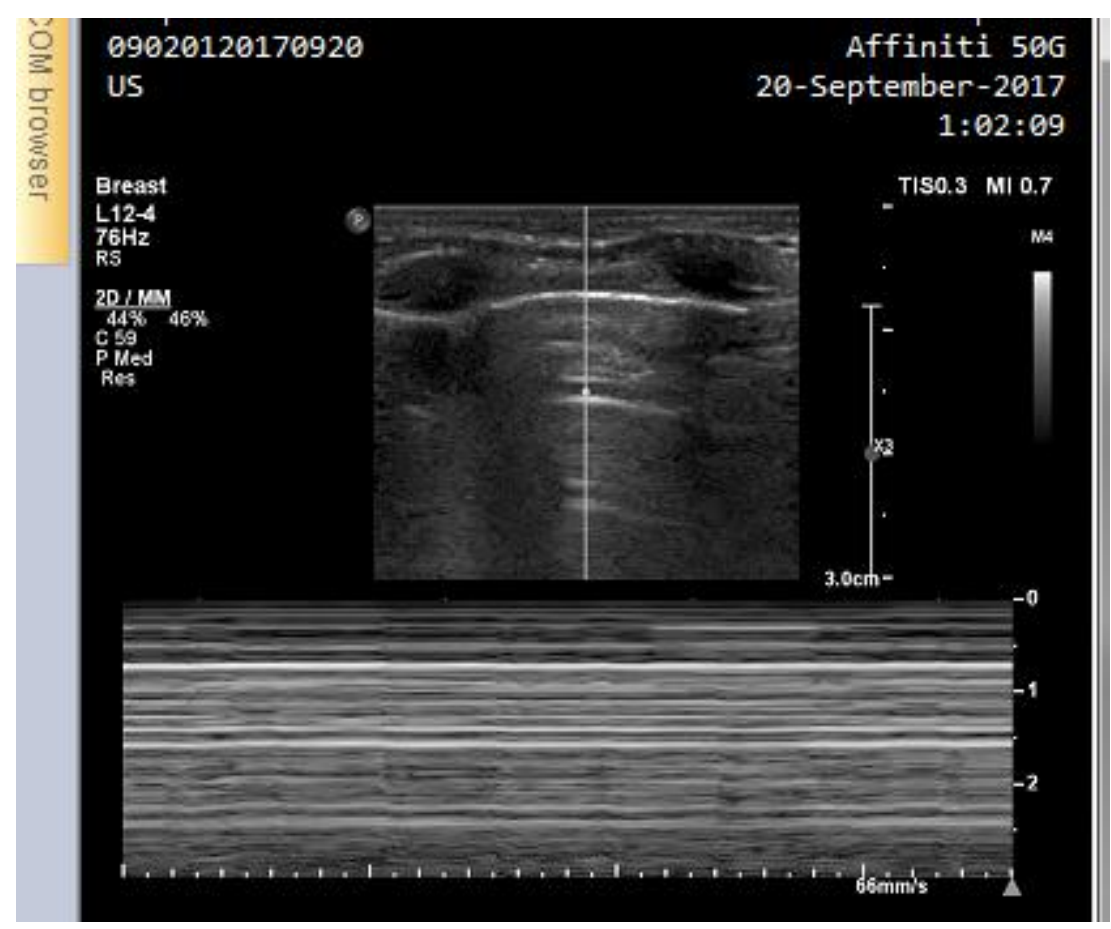

In conclusion, lung ultrasound done by emergency physician has high accuracy in diagnosis of pneumonia presented with acute respiratory failure. And when clinical examination of those patients added to the results of lung ultrasound, the accuracy may be even higher.

\section{Acknowledgements}

Prof. Mohamed Elhenaidy head of emergency medicine department. Prof. Hala M. Elgendy Prof of anesthesia and intensive care for her support. Dr. Wesam Ibrahim and Dr. Mohamed Bosily for their effort in training emergency physicians on lung ultrasound.

Funding: The author(s) received no financial support for the research, authorship, and/or publication of this article.

Ethical approval: Ethical approval for the study was obtained from ethical committee of Tanta University Faculty of Medicine before the start of the research.

\section{References}

1. Vincent J-L, Akça S, De Mendonça A, Haji-Michael P, Sprung C, Moreno R, et al., The epidemiology of acute respiratory failure in critically ill patients. Chest. 2002;121(5):1602-9.

2. Bouhemad B, Zhang M, Lu Q, Rouby JJ. Clinical review: bedside lung ultrasound in critical care practice. Critical Care. 2007;11(1):205.

3. Maffessanti M, Berlot G, Bortolotto P. Chest roentgenology in the intensive care unit: an overview. European radiology. 1998;8(1):69-78.

4. Moore CL, Copel JA. Point-of-care ultrasonography. New England Journal of Medicine. 2011;364(8):749-57.

5. Zanobetti M, Scorpiniti M, Gigli C, Nazerian P, Vanni S, Innocenti F, et al., Point-of-care ultrasonography for evaluation of acute dyspnea in the ED. Chest. 2017; 151(6):1295-301.

6. Lichtenstein DA. Lung ultrasound in the critically ill. Annals of intensive care. 
2014;4(1):1.

7. Pivetta E, Goffi A, Lupia E, Tizzani M, Porrino G, Ferreri E, et al., Lung ultrasound-implemented diagnosis of acute decompensated heart failure in the ED. Chest. 2015; 148(1): 202-10.

8. Lichtenstein DA, Mezière GA. Relevance of lung ultrasound in the diagnosis of acute respiratory failure: the BLUE protocol. Chest. 2008; 134.

9. Cortellaro F, Colombo S, Coen D, Duca PG. Lung ultrasound is an accurate diagnostic tool for the diagnosis of pneumonia in the emergency department. Emerg Med J. 2012; 29(1): 19-23.

10. Abdalla W, Elgendy M, Abdelaziz A, Ammar M. Lung ultrasound versus chest radiography for the diagnosis of pneumothorax in critically ill patients:
A prospective, single-blind study. Saudi Journal of Anaesthesia. 2016;10(3):265.

11. Mumtaz U, Zahur Z, Chaudhry MA, Warraich RA. Bedside Ultrasonography: A Useful Tool for Traumatic Pneumothorax. Journal of the College of Physicians and Surgeons-Pakistan: JCPSP. 2016; 26(6): 459-62.

12. Zhang M, Liu ZH, Yang JX, Gan JX, $\mathrm{Xu}$ SW, You XD, et al., Rapid detection of pneumothorax by ultrasonography in patients with multiple trauma. Critical care (London, England). 2006; 10(4): R112.

13. Rowan KR, Kirkpatrick AW, Liu D, Forkheim KE, Mayo JR, Nicolaou S. Traumatic pneumothorax detection with thoracic US: correlation with chest radiography and CT--initial experience. Radiology. 2002; 225(1): 210-4.

\section{How to cite this article:}

Mohamed Elbahnasawy, Ghada Atef Attia, Atef Hamad Teima and Abdel Hady Mohamed Taha. 2019. The Accuracy of Barcode Sign in Diagnosis of Pneumothorax Associated with Acute Respiratory Failure in Emergency Department. Int.J.Curr.Microbiol.App.Sci. 8(06): 989994. doi: https://doi.org/10.20546/ijcmas.2019.806.120 